\title{
Guidelines for the treatment of rheumatic patients in the pandemic of COVID-19 infection
}

\author{
Mansoor Karimifar $^{1 *(1)}$, Mozhgan Karimifar ${ }^{2}$ \\ 'Departments of Rheumatology, Alzahra Hospital, Isfahan University of Medical Sciences Isfahan, Iran \\ ${ }^{2}$ Isfahan Endocrine and Metabolism Research Centre, Isfahan University of Medical Sciences, Isfahan, Iran
}

\author{
Correspondence to: \\ Mansoor Karimifar \\ Email:mansoor_karimifar@ \\ yahoo.com
}

Received: 14 Sep. 2020

Accepted: 8 Dec. 2020

ePublished: 27 Dec. 2020

Citation: Karimifar M, Karimifar M. Guidelines for the treatment of rheumatic patients in the pandemic of COVID-19 infection. J Prev Epidemiol. 2020;5(2):e30. doi: $10.34172 /$ jpe. 2020.30

\author{
Key point \\ Cytokine storm may occur in COVID-19 patients, and this phenomenon is similar to rheumatic disease. \\ Keywords: COVID-19, Rheumatoid arthritis, Lupus, Wegener's granulomatosis, Polymerase chain reaction
}

A round $20 \%$ of patients with COVID-19 develop lung involvement, of which $5 \%$ go to respiratory failure and multiple organ involvement, and $1 \%$ of them die. During the second week of the disease, a phenomenon called cytokine storm may occur in these patients, which is accompanied by a decrease in CD4, CD8 lymphocytes and interferons I and III, an increase in proinflammatory cytokines such as interleukin-1 (IL-1), IL-6, tumour necrosis factor alpha (TNF alpha) and granulocyte colony-stimulating factor (G-CSF), and finally T-cell fatigues. In the cytokine storm, the patient develops fever and increased C-reactive protein (CRP), ferritin and D-dimer. Occasionally the patient develops anemia due to the onset of macrophage activation syndrome. Because these laboratory changes are common in rheumatic patients, the idea of using antirheumatic drugs in the treatment of patients with COVID- 19 emerged, although they could potentially spread the infection (1).
Target therapy
Today, the treatment of rheumatic diseases is moving towards targeted therapy, because it targets only one specific cytokine and does not inhibit innate and adaptive immunity, whereas cytotoxic drugs are not. One of the oldest targets for rheumatology is anti- TNF-alpha drugs. Anti-TNF-alpha drugs can cause severe bacterial infections, but there has been little evidence of worsening influenza with these drugs. Rituximab, an anti-CD20 drug, mainly reduces humoral immunity. Newer target therapies such as

guselkumab, which are anti-IL-23, and antiIL-4, anti-IL-13, and anti-IL-17A drugs, have not been associated with an increased risk of viral infections.

Small molecules (tsDMARDs; targeted synthetic disease-modifying antirheumatic drugs) such as tofacitinib, on one hand, inhibit interferon I, IL-2, IL-15, IL-21, and gamma interferon, which are vital in controlling infections; on the other hand, they prevent the release of proinflammatory mediators such as IL-17, IL-6, GM-CSF and prevents the virus from entering cells, so it has a dual role and it is not clear which ones will prevail if is used in COVID-19 patients.

Hydroxychloroquine (HCQ) is an excellent modulator of the immune system and does not cause the spread of infection. Contradictory studies against this drug are due to its concomitant use with other drugs that increase the QT interval and arrhythmia, but if used properly, it is good medicine. There is insufficient information on the use of azathioprine (AZA), methotrexate (MTX), cyclosporine, and mycophenolate mofetil (MMF) in the treatment of cytokine storm COVID-19 $(2,3)$.

\section{Glucocorticoids}

At the beginning of the coronavirus epidemic, it was recommended to use them only in mechanically ventilated patients, but now it is used in moderate to high doses in all hospitalized patients $(4,5)$.

Prognosis of COVID-19 in men and women

Various studies have shown that being a 
woman, is a protective factor against coronavirus mortality and rheumatic patients are no exception to this rule (6).

Hospitalization rate of rheumatic patients with COVID-19

An American study of rheumatic patients with COVID-19 found that the rate of hospitalization was no different from the general population (7).

In a small study of lupus patients in France, they concluded that hydroxychloroquine did not prevent severe COVID-19 (8), but the disadvantage of this study was that comorbidities such as diabetes, obesity, old age, and hypertension, heart failure, kidney failure and chronic lung disease, were not considered. Another Italian study of rheumatic patients found similar results to the American study (9).

\section{Case Reports}

In one case report, a 52-year-old woman with Wegener who was treated with rituximab and prednisolone developed COVID-19 disease. After admission, the patient was treated with the antiviral drug Kaletra and hydroxychloroquine, but due to respiratory failure was mechanically ventilated and removed from the ventilator after 2 days (10). The authors concluded that this rapid recovery was due to the use of rituximab and prednisolone. In another case report in the UK, a 23-year-old man with Crohn's disease developed COVID-19 and did not need to be hospitalized because he was taking the anti-TNF-alpha (infliximab) drug (11).

In another case report, a woman with ulcerative colitis treated with Tofacitinib did not need to be admitted (11).

In another report, a 53-year-old woman with Crohn's disease who was being treated with adalimumab needed to be hospitalized, but the patient was not mechanically ventilated (11).

Based on the above evidence, it cannot be said at this time that the use of DMARDs prevents severe COVID-19. Additionally, according to this evidence, the risk of COVID-19 in rheumatic patients is not higher than the general population $(9,12)$.

Similarity of symptoms of rheumatic diseases with COVID-19 and vice versa

Weakness, myalgia and fatigue are the three most common symptoms in both rheumatic and COVID-19 disease, so diagnosing flare-up of rheumatic disease from COVID-19 requires a laboratory test. Fever is also present in some rheumatism, such as lupus, and in COVID-19, so in nonrheumatic patients, attention to both aspects is essential and the diagnosis of rheumatic disease should not be the victim of a COVID-19 infection. Headaches are seen in both COVID-19 and giant cell arteritis. Gastrointestinal symptoms can also be due to COVID-19 or complications from the use of certain anti-rheumatic drugs such as sulfasalazine or following spondyloarthritis, scleroderma (systemic sclerosis), lupus or Behcet's syndrome. Dyspnea is found in both COVID-19 and interstitial lung disease due to rheumatoid arthritis (RA), systemic lupus erythematosus and systemic sclerosis. Vascular events are seen in both COVID-19 and antiphospholipid syndrome. There are many types of skin lesions in both the COVID-19 and rheumatic diseases. Children with COVID-19 have occasionally reported symptoms similar to Kawasaki disease. A physician must make a number of laboratory test abnormalities are seen in both COVID-19 and rheumatic diseases, such as increased erythrocyte sedimentation rate (ESR), CRP, ferritin, IL-6, and CPK, and the distinction between the two. Drug side effects should also be differentiated from COVID-19. For example, sometimes pulmonary side effects are seen following MTX. Sulfasalazine may also cause gastrointestinal disorders (13).

\section{Emergence of rheumatic diseases after COVID-19}

A case report of a woman with COVID-19 who had recently recovered, developed high-titer of anti-CCP antibodies and arthritis, and the diagnosis of RA was made, and treated with MTX. It has been hypothesized that COVID-19 infection causes epitope spread and RA (14).

Small coronary vasculitis following COVID-19 has also been reported, with another report showing IgA-like Henoch-Schönlein purpura vasculitis in a Crohn's disease patient. In another report, reactive arthritis was seen in a patient following COVID-19 and had no underlying disease before $(15,16)$.

Management of drugs in rheumatic patients with asymptomatic positive PCR test for COVID-19

If the patient has been using HCQ, chloroquine, SSZ (sulfasalazine), or nonsteroidal anti-inflammatory drugs (NSAIDs), such drugs are not at risk of spreading the infection and will continue unless there is a specific contraindication, for example in acute renal failure, NSAIDs should be discontinued.

MTX, cyclophosphamide, MMF (mycophenolic acid), AZA, tacrolimus, anti-TNF-alpha drugs, abatacept, antiIL-6 drugs, JAK inhibitors such as Tofacitinib, should be discontinued for 2 weeks after a positive polymerase chain reaction (PCR) test or the PCR test becomes negative again (17).

Drug management of rheumatic patients with symptomatic COVID-19 and positive PCR test

Continue antimalarial drugs; discontinue SSZ, MTX, MMF, AZA, Biologic agent, and Tofacitinib during active COVID-19. The resumption of these drugs varies depending on the general condition of the patients.

Prednisolone should be continued in patients with COVID-19 because it prevents both the onset of rheumatic 
disease and the adrenal insufficiency (17).

Time to restart antirheumatic drugs after COVID-19 infection

In asymptomatic patients with a positive PCR test 10 to 17 days after the positive PCR test and in symptomatic patients without complications 7 to 14 days after improvement of symptoms, medications can be restarted. In severe and complicated patients, decide to start the medication again for each patient individually, depending on the general condition (17).

\section{Seroconversion}

This condition usually occurs 2 weeks after the illness and the patient is no longer contagious. In some cases, a positive PCR test remains positive for up to 30 days and causes concern to those around the patient, but research has shown that after 8 days The matte test, even if the PCR test is positive again, is due to the dead, non-cultured pieces of the virus and does not pose a risk to those around the patient. Another point is that if the patient remains asymptomatic for 3 days, it can be said that the person is immune to the disease and has antibodies.

\section{Conclusion}

Finally, the onset of rheumatic disease after recovery of covid-19 is not common, although there are case reports in this area (17).

\section{Authors' contribution}

MaK wrote the primary draft. MoK edited the final manuscript. All authors read and signed the final paper.

Conflicts of interest

The authors declare that they have no competing interests.

Ethical considerations

Ethical issues (including plagiarism, data fabrication, double publication) have been completely observed by the authors.

\section{Funding/Support}

None.

\section{References}

1. Vardhana SA, Wolchok JD. The many faces of the anti-COVID immune response. J Exp Med. 2020;217:e20200678. doi: 10.1084/jem.20200678.

2. Singh JA, Cameron C, Noorbaloochi S, Cullis T, Tucker M, Christensen $\mathrm{R}$, et al. Risk of serious infection in biological treatment of patients with rheumatoid arthritis: a systematic review and meta-analysis. Lancet. 2015;386:258-65. doi: 10.1016/S0140-6736(14)61704-9.

3. Dirven L, Huizinga TW, Allaart CF. Risk factors for reported influenza and influenza-like symptoms in patients with rheumatoid arthritis. Scand J Rheumatol. 2012;41:359-65. doi: 10.3109/03009742.2012.670729.

4. Bhimraj A, Morgan RL, Shumaker AH, Lavergne $\mathrm{V}$, Baden
L, Cheng VC, et al. Infectious Diseases Society of America Guidelines on the Treatment and Management of Patients with COVID-19. Clin Infect Dis. 2020:ciaa478. doi: 10.1093/cid/ ciaa478.

5. Russell CD, Millar JE, Baillie JK. Clinical evidence does not support corticosteroid treatment for 2019-nCoV lung injury. Lancet. 2020;395:473-475. doi: 10.1016/S01406736(20)30317-2

6. de Groot NG, Bontrop RE. COVID-19 pandemic: is a genderdefined dosage effect responsible for the high mortality rate among males? Immunogenetics. 2020;72:275-277. doi: 10.1007/s00251-020-01165-7.

7. Haberman R, Axelrad J, Chen A, Castillo R, Yan D, Izmirly P, et al. Covid-19 in Immune-mediated inflammatory diseases case series from New York. N Engl J Med. 2020;383:85-88. doi: 10.1056/NEJMc2009567.

8. Mathian A, Mahevas M, Rohmer J, Roumier M, Cohen-Aubart $\mathrm{F}$, Amador-Borrero B, et al. Clinical course of coronavirus disease 2019 (COVID-19) in a series of 17 patients with systemic lupus erythematosus under long-term treatment with hydroxychloroquine. Ann Rheum Dis. 2020;79:837-839. doi: 10.1136/annrheumdis-2020-217566.

9. Monti S, Balduzzi S, Delvino P, Bellis E, Quadrelli VS, Montecucco C. Clinical course of COVID-19 in a series of patients with chronic arthritis treated with immunosuppressive targeted therapies. Ann Rheum Dis. 2020;79:667-668. doi: 10.1136/annrheumdis-2020-217424.

10. Guilpain P, Le Bihan C, Foulongne V, Taourel P, Pansu N, Maria ATJ, et al. Rituximab for granulomatosis with polyangiitis in the pandemic of covid-19: lessons from a case with severe pneumonia. Ann Rheum Dis. 2021;80:e10. doi: 10.1136/ annrheumdis-2020-217549.

11. Price E, MacPhie E, Kay L, Lanyon P, Griffiths B, Holroyd $C$, et al. Identifying rheumatic disease patients at high risk and requiring shielding during the COVID-19 pandemic. Clin Med (Lond). 2020:clinmed.2020-0160. doi: 10.7861/ clinmed.2020-0160.

12. Favalli EG, Agape E, Caporali R. Incidence and Clinical Course of COVID-19 in Patients with Connective Tissue Diseases: A Descriptive Observational Analysis. J Rheumatol. 2020;47:1296. doi: 10.3899/jrheum.200507.

13. Hsu CY, Ko CH, Wang JL, Hsu TC, Lin CY. Comparing the burdens of opportunistic infections among patients with systemic rheumatic diseases: a nationally representative cohort study. Arthritis Res Ther. 2019;21:211. doi: 10.1186/ s13075-019-1997-5.

14. Perrot L, Hemon M, Busnel JM, Muis-Pistor O, Picard C, Zandotti $C$, et al. First flare of ACPA-positive rheumatoid arthritis after SARS-CoV-2 infection. Lancet Rheumatol. 2021;3:e6-e8. doi: 10.1016/S2665-9913(20)30396-9.

15. Fox SE, Lameira FS, Rinker EB, Vander Heide RS. Cardiac Endotheliitis and multisystem inflammatory syndrome after COVID-19. Ann Intern Med. 2020;173:1025-7. doi: 10.7326/ L20-0882.

16. Allez M, Denis B, Bouaziz JD, Battistella M, Zagdanski AM, Bayart J, et al. COVID-19-Related IgA Vasculitis. Arthritis Rheumatol. 2020;72:1952-1953. doi: 10.1002/art.41428.

17. Mikuls TR, Johnson SR, Fraenkel L, Arasaratnam RJ, Baden LR, Bermas BL, et al. American College of Rheumatology Guidance for the Management of Rheumatic Disease in Adult Patients During the COVID-19 Pandemic: Version 1. Arthritis Rheumatol. 2020;72:1241-1251. doi: 10.1002/art.41301. 